\title{
Verbot trotz Bedeutungslosigkeit? Die NPD, ihre Strategie und die Erfolgsaussichten des neuen Verbotsantrags
}

\author{
Michael Oswald
}

Die Nationaldemokratische Partei Deutschlands (NPD) feiert in diesem Jahr ihr 50-jähriges Bestehen. Gründe zum Feiern haben ihre Funktionäre jedoch kaum: Die Partei ist hoch verschuldet, ihre Mitgliederzahl rückläufig, die Prognosen für die Landtagswahlen stehen gegen die Partei, und mit Blick auf ein abermaliges Parteiverbotsverfahren birgt 2014 keine guten Aussichten. Zudem hat der Richtungsstreit zwischen bürgerlicher Annäherung und Inklusion der extremen Rechten nicht nur zwei Vorsitzende verschlissen, sondern auch die Partei mehr zerrüttet denn je. An die politischen Erfolge der Vergangenheit konnte zudem nicht angeknüpft werden. Nicht wenige Analysten sehen die NPD nicht nur angeschlagen, sondern bereits unfähig, noch einmal aufzustehen. In der Presse wurde bisweilen gemutmaßt, sie läge bereits im Sterben. ${ }^{1}$

Seit mehr als vier Dekaden wird über ein Verbot der NPD diskutiert, aber bis heute ist strittig, ob dies auch sinnvoll sei: Einen Tag bevor der aktuelle Verbotsantrag beim Bundesverfassungsgericht eingereicht wurde, teilte der Sprecher der Bundesregierung, Steffen Seibert mit, dass die Bundesregierung zwar das Vorgehen des Bundesrats unterstütze, eine eigene Initiative jedoch nicht für erforderlich halte. ${ }^{2}$ In der Antragsschrift wollen die Vertreter des Bundesrats, Christian Waldhoff und Christoph Möllers, nachweisen, dass die NPD die Beseitigung der freiheitlich demokratischen Grundordnung nach Artikel 21 Abs. 2 GG anstrebt ${ }^{3}$; ob dies jedoch als aktive Maßnahme gewertet werden kann, bleibt zunächst offen. Auch wenn die Autoren auf Beweise verzichteten, die von V-Männern oder verdeckten Ermittlern stammen könnten, garantiert dies allein noch kein erfolgreiches Verfahren.

Vor allem fällt es schwer, der NPD politische Wirksamkeit nachzuweisen - eine dringende Notwendigkeit für ihr Verbot. Zwar argumentieren die Prozessvertreter richtig, dass die Partei eindeutige Wesenszüge der NSDAP enthält. Doch anders als die NSDAP, die ein junges und instabiles Regierungssystem mit der Rückendeckung der Massen bekämpfen konnte und ihre Gegner mittels Schlägertrupps verängstigte, ist die NPD in einem desolaten Zustand und von der großen Mehrheit der Bürger geächtet. Deshalb spricht viel dafür, dass der Europäische Gerichtshof für Menschenrechte ein Verbot kassieren würde, weil die Demokratie in Deutschland nicht im Zugzwang scheint, mit ihrer schärfsten Waffe gegen Rechtsextremisten vorzugehen, die ohnehin kaum Einfluss haben.

Das Verbotsverfahren ist auf der moralischen Ebene verständlich: Deutschland will sich zu seinen kulturellen Werten bekennen und nach den schrecklichen Morden des NSU zei-

1 Vgl. Andreas Förster, Die NPD ist eine sterbende Partei, in: FR online vom 4. Dezember 2012, http://www.fr-online.de/politik/npd-die-npd-ist-eine-sterbende-partei, 1472596,21035138.html (Abruf am 15. Januar 2014).

2 Zu den Verfahrensfortschritten und den jeweils aktuellen Verfahrensstand informiert der Bundesrat auf seiner Homepage, http://www.bundesrat.de/static/Web/DE/presse/Thema/Themanode__nnn-true.html (Abruf am 12. Januar 2014).

3 Vgl. Christoph Möllers / Christian Waldhoff, Antrag nach Art. 21 Abs. 2 GG i.V.m. \$\$ 13 Nr. 2, 43 ff. BVerfG, S. 11, S. 16- 18. 
gen, dass in diesem Land kein Platz für Rechtsextremismus ist. Aus dieser Sicht ist die Symbolik und Richtung des Verbotsantrags zweifelsohne richtig; aus politikwissenschaftlicher Perspektive wandelt sich die Entscheidung des Bundesverfassungsgerichtes damit jedoch eher zu einer Gretchenfrage der Politischen Kultur in Deutschland.

\section{Die NPD: Eine extremistische Partei}

Die NPD ist in der Tat eine extremistische Partei, ohne Zweifel sind die Mitglieder der NPD extremistisch eingestellt. Hierüber besteht Konsens in Politik, Publizistik und Politikwissenschaft. ${ }^{4}$ Letztere definiert den Extremismus-Begriff allerdings nicht einheitlich: Er beschreibt diverse Phänomene und wird in verschiedenen Forschungsfeldern verwendet. Als allgemein akzeptiert gilt, dass Extremismus nur in Abhängigkeit von Norm- oder Wertvorstellungen zu verstehen ist. In dieser Definition stehen Extremisten fundamentalen Werten der Demokratie entgegen - in erster Linie der freiheitlichen demokratischen Grundordnung. Praktisch fallen darunter „politische Diskurse, Programme und Ideologien [...], die sich implizit oder explizit gegen grundlegende Werte und Verfahrensregeln demokratischer Verfassungsstaaten richten"5. Dies gilt vor allem, wenn die Demokratie durch eine politische Ordnung mit totalitären Zügen ersetzt werden soll.

Etymologisch gründet der Begriff auf eine Position, die sich an äußerster Stelle befindet. In Bezug auf eine hypothetische „Mitte der Gesellschaft“, die für eine Form von „Political Correctness" und gesellschaftlichen „Common Sense“ steht, ist sie damit als eine äußerste Abweichung zu verstehen. ${ }^{6}$

Mangels einer exakten Definition des Extremismus-Begriffs verfügt die Politikwissenschaft über keine allgemeine Klassifikation für extremistische Parteien. Nach der genannten Negativdefinition wäre ihr Hauptmerkmal das Ziel, nach einer "Machtübernahme“ einen Staat mit autoritären oder totalitären Zügen zu errichten. ${ }^{7}$ In jedem Fall würden dabei bestimmte wesensrelevante Elemente der Demokratie beseitigt, Gruppen von Menschen geächtet oder gar aus der Gesellschaft ausgeschlossen.

Anhänger extremistischer Parteien vertreten ein geschlossenes politisches Weltbild und verabsolutieren ihre Ideologie, der sie in einer prinzipiengebundenen, starren Denk- und Handlungsweise folgen; ihre Weltanschauung wird kaum reflektiert oder fortentwickelt. Außerdem gilt sie ihnen als absolute Wahrheit. ${ }^{8}$ Diesem Anspruch entspringt ein Sendungsbewusstsein, während die Legitimität unterschiedlicher Meinungen und Interessen auf ein Minimum beschnitten wird. ${ }^{9}$ Darum stehen Extremisten dem Pluralismus entgegen

4 Vgl. Eckhard Jesse, Die Diskussion um ein neuerliches NPD-Verbotsverfahren - Verbot: kein Gebot, Gebot: kein Verbot, in: ZfP, 50. Jg. (2012), H. 3, S. 296 - 313, S. 303.

5 Uwe Backes, Gestalt und Bedeutung des intellektuellen Rechtsextremismus in Deutschland, in: APuZ, B 46/2001, S. 24 - 30, S. 24.

6 Vgl. Eckhard Jesse, Formen des politischen Extremismus, in: Bundesministerium des Innern (Hrsg.), Extremismus in Deutschland. Erscheinungsformen und aktuelle Bestandsaufnahme, Berlin 2004, S. 7 - 24, S. 10.

7 Vgl. ebenda, S. 10.

8 Vgl. Hans-Gerd Jaschke, Politischer Extremismus, Wiesbaden 2006, S. 31, S. 36, S. 85.

9 Vgl. Eckhard Jesse, a.a.O. (Fn. 4), S. 11. 
und verachten inhaltliche Diskussionen. Politik ist für sie letztendlich keine Diskussion um politische Inhalte, sondern eine Weltanschauung, die auf ein allgemeines Freund-FeindDenken gründet. Kompromisse oder Konsens sind daher keine politische Option für Extremisten.

Basierend auf einer Strategie, die eine Machtübernahme über Wahlen möglich machen soll, im Verbund mit entsprechenden Aussagen wie jene des ehemaligen Parteichefs Udo Voigt, dass es sein Ziel sei, die Bundesrepublik über die Wahlurne „abzuwickeln“"10, muss die NPD als ein Typus des „systemimmanenten Rechtsextremismus“ eingeordnet werden. Extremistische Parteien können nach Artikel 21 GG nur durch das Bundesverfassungsgericht verboten werden. Das BVerfG hat sich allerdings selbst - zum Schutze demokratischer Verfahren - eine Hürde auferlegt: Es muss nachgewiesen werden, dass eine verfassungsfeindliche Partei auch eine kämpferisch-aggressive Haltung gegen die bestehende politische Ordnung an den Tag legt.

Für den Juristen Christian Hufen lauern in der Verbotsforderung bereits „vernachlässigte Bedenken, ob es gelingen kann, der NPD die Verfassungswidrigkeit nach Artikel 21 II GG tatsächlich nachzuweisen“11. Er begründet seine skeptische Haltung damit, dass die „Aneinanderreihung extremistischer Äußerungen der Parteimitglieder und ihre Gewaltbereitschaft [...] dafür nicht aus[reicht]; es müssen aktive Maßnahmen gegen die freiheitliche demokratische Grundordnung in Angriff genommen werden"12. Nach Eckhard Jesse kann zwar die Strategie der NPD bereits als kämpferisch-aggressiv ausgelegt werden ${ }^{13}$; diese vermochte es jedoch nicht, die politische Ordnung Deutschlands zu verändern. Dem Europäischen Gerichtshof für Menschenrechte zufolge muss aber ein Parteiverbot gesellschaftlich dringend notwendig sein. Dies sieht Armin Pfahl-Traughber nicht gegeben: Seine Auswertung juristischer und politischer Aspekte eines etwaigen Verbots führt „zu einer ablehnenden Auffassung, würde das ,scharfe Schwert‘ eines solchen Schrittes doch gegenüber einer gesamtgesellschaftlich kaum bedeutsamen politischen Kraft eingesetzt "14. Wie ist es also um die gesellschaftliche Wirksamkeit der NPD heute bestellt?

\section{Die Strategie, die Unmögliches leisten soll}

Die im Folgenden diskutierte Strategie wird aufgrund ihrer anfänglichen Erfolge lange vom Führungskader forciert. In $₫ 3$ der Parteisatzung ist das Ziel der NPD festgeschrieben: Sie wolle „die politische Wirksamkeit in allen Teilen Deutschlands“15 erreichen. Dies sollte

10 Vgl. Udo Voigt, Der NPD-Vorsitzende Udo Voigt über den Wahlerfolg seiner Partei und den „Zusammenbruch des liberal-kapitalistischen Systems“, in: Junge Freiheit vom 24. September 2004, S. 3.

11 Christian Hufen, Neues Parteiverbotsverfahren gegen die NPD? Verfassungsrechtliche Voraussetzungen vor dem Hintergrund der NSU Taten, in: ZRP, 45. Jg. (2012), H. 7, S. $202-205$, S. 202.

12 Ebenda.

13 Vgl. Eckhard Jesse, a.a.O. (Fn. 4), S. 304.

14 Vgl. Armin Pfahl-Traughber, Kann und soll man die NPD verbieten?, in: Humanistischer Pressedienst vom 7. Dezember 2012, http://hpd.de/node/14549 (Abruf am 26. Juni 2013).

15 NPD, Satzung 06/2012, http://www.bundeswahlleiter.de/de/parteien/downloads/parteien/Nationaldemokratische_Partei_Deutschlands.pdf (Abruf am 17. Mai 2013). 
langfristig auf Basis des 1998 lancierten Drei-Säulen-Konzeptes erreicht werden - der „Kampf um die Köpfe“, der „Kampf um die Straße“ und der „Kampf um die Wähler“. Nach den Wahlerfolgen von 2004 in Sachsen wurden die Grundpfeiler ihres politischen Wirkens um eine weitere Säule ergänzt: den „Kampf um den organisierten Willen“. Dieser wurde notwendig, um einen personellen Mangel mit der Hilfe sämtlicher rechter Strömungen zu kompensieren. Die offene Zusammenarbeit mit gewaltbereiten Neonazis war durch das Scheitern des Parteiverbotsverfahrens von 2003 möglich geworden, das als eine Art Freibrief aufgefasst wurde.

\subsection{Die Intellektualisierung im Rechtsradikalismus}

Der „Kampf um die Köpfe“ war als ein Intellektualisierungsprozess gedacht, der die Argumentationsgrundlagen für die Parteiarbeit theoretisch untermauern und dazu beitragen sollte, sich vom dumpfen Rechtsradikalismus zu lösen. Zu diesem Zweck wurde unter anderem im Jahre 2004 die Dresdner Schule als „Denk- und Politikschule einer selbstbewußten [sic!] Nation "16 gegründet. Der im Selbstverständnis als Gegenbewegung zur Frankfurter Schule geschaffene ,Think Tank' sollte eine „geistig-politische Front [...] gegen die volks- wie staatszersetzende BRD-Nomenklatura [...] aufbauen "17. Das Bildungswerk für Heimat und nationale Identität e.V. dient einem ähnlichen Zweck und versteht sich als Stätte für politische Bildungsarbeit. Sie nimmt die Funktion einer parteinahen Stiftung ein, „die den Wertvorstellungen der Nationaldemokratischen Partei Deutschlands nahesteht"18. Beide Institutionen sollen die Intellektualisierung im Umkreis der NPD vorantreiben.

Die Urheber dieses Kurses waren zunächst die NPD-Kader Karl Richter und Jürgen Gansel - sie fungierten bei jener Entwicklung als „Ideologen“. Diese Denkweise hat sich nunmehr auf den gesamten Wirkungskreis um Gansel ausgedehnt. ${ }^{19}$ Dies ist vor allem in Veröffentlichungen zu erkennen: Das Bildungswerk ist Herausgeber der Zeitschrift „hier \& jetzt“, die eine „wohldosierte Mischung aus Theorietexten, profunden Analysen aktueller oder historischer Vorgänge "20 anpreist. Dabei versuchen ihre Autoren wissenschaftliche Erkenntnisse und Elemente der Theoretiker der Konservativen Revolution in das rechte Ideologiespektrum einfließen zu lassen. ${ }^{21}$ Ernst Jünger ist ein stetig wiederkehrender Name in den Publikationen. In der Tat birgt jene Strömung des antidemokratischen Denkens der Weimarer Republik das Potenzial, die Grenzen zu konservativen Bewegungen zu verwäs-

16 Jürgen Gansel, Erklärung des Landtagsabgeordneten zu Wesen und Wollen der „Dresdner Schule“. Frankfurt war gestern, Dresden ist heute - Denk- und Politikschule einer selbstbewußten Nation, http://www.npd.de/index.php?pdfdruck=1\&pfad_id=9\&cmsint_id=1\&detail=291\&vid =1520.pdf (Abruf am 4. Juni 2013).

17 Ebenda.

18 Bildungswerk, Bildungswerk für Heimat und nationale Identität, http://heimat-bildungswerk.de (Abruf am 19. April 2014).

19 Die Vorstände des Bildungswerkes (Thorsten Thomsen, Holger Szymanski, Frank Ahrens und Arne Schimmer, der Chefredakteur von ,hier \& jetzt“) sind allesamt Funktionäre der NPD Sachsen.

20 Bildungswerk, 168 Seiten rechte Metapolitik aus der Dresdner Theoriewerkstatt, http://heimatbildungswerk.de/2012/04/17/168-seiten-rechte-metapolitik-aus-der-dresdner-theoriewerkstatt/ (Abruf am 19. April 2014).

21 Vgl. Armin Pfahl-Traughber, Der zweite Frühling der NPD, Sankt Augustin 2008, S. 42 f. 
sern und rechtsextreme Ideen auf Basis eines wissenschaftlichen Hintergrunds legitim wirken zu lassen. ${ }^{22}$

Die Akteure adaptierten damit im Grunde ein Konzept der Neuen Rechten (NR). Die NR versteht sich als Avantgarde der rechten Bewegung, die als Bindeglied zwischen Konservatismus und intellektuellem Rechtsextremismus fungieren will. Sie ist hauptsächlich in publizistischen Projekten aktiv und prägt so sämtliche rechte Strömungen - von den Kolumnen der Jungen Freiheit bis hin zum Parteiprogramm der NPD. ${ }^{23}$

Die Denker der NR sehen sich selbst als Rechtsintellektuelle und können in zwei Hauptgruppen eingeteilt werden: eine, die ihre Ansichten fundamentalistisch äußert und eine zweite, die sie in Form von Mimikry zurückhält. Exemplarisch kann für letztere Variante das im Jahr 2000 gegründete Institut für Staatspolitik und ihr publizistisches Organ, die Zeitschrift Sezession, gelten - sie fungiert als Denkfabrik der NR. Seine Gründer Karlheinz Weißmann und Götz Kubitschek empfehlen, dass man unter ungünstigen politischen Rahmenbedingungen die Mimikry-Strategie nutzen solle. ${ }^{24}$ Diesem Ratschlag folgen viele NPD-Funktionäre. Mimikry wurde zu einem der wichtigsten Punkte des öffentlichen Agierens: In Schulungen wurde Kadern Rhetorik und Strategiefähigkeit vermittelt, dazu zählen auch die Entsagung von Gewalt und ein Auftreten, das einen NPD-Aktivisten äußerlich nicht mehr vom „Normalbürger“ unterscheidet. $^{25}$ Ein angepasstes und adäquates Erscheinungsbild sei wichtig, um die Gesellschaft nicht abzuschrecken.

\section{2. Öffentlichkeitswirksamkeit als strategischer Grundsatz}

Da die NPD als extremistische Partei weder positive Medienunterstützung noch breiten Zuspruch aus der Gesellschaft erfährt, muss sie sich strategisch in der Öffentlichkeit platzieren, um Beachtung zu erfahren. ${ }^{26}$ Dafür sorgte lange der „Kampf um die Straße“. Die Straße dient der NPD bereits seit den späten 90er Jahren als öffentlichkeitswirksamer politischer Raum; mittels häufiger und groß angelegter Demonstrationen wollte sie sich der Gesellschaft zeigen und ihren Machtanspruch präsentieren. ${ }^{27}$ Die Aktionsform der Demonstration wurde von jener Zeit an geradezu zelebriert und zu einem wichtigen Bestandteil für ihr öffentliches Wirken. Doch von der bloßen Vertretung einer bestimmten sozialen Gruppierung wollte die NPD strategisch abrücken. Ziel war es, vor allem Menschen außer-

22 Vgl. Hans-Gerd Jaschke, a.a.O. (Fn. 8), S. 85.

23 Vgl. Thomas Pfeiffer / Michael Puttkamer, Warum das Land Nordrhein-Westfalen die Junge Freiheit in seinen Verfassungsschutzberichten geführt hat, in: Stephan Braun / Ute Vogt (Hrsg.), Die Wochenzeitung Junge Freiheit. Kritische Analysen zu Programmatik, Inhalten, Autoren und Kunden, Wiesbaden 2007, S. 57 - 76, S. 60.

24 Armin Pfahl-Traughber, a.a.O. (Fn. 21), S. 57 f.

$25 \mathrm{Vgl}$. David Begrich / Thomas Weber, Was meint die Taktik der Wortergreifung, in: Fabian Virchow / Christian Dornbusch (Hrsg.), 88 Fragen und Antworten zur NPD, Schwalbach 2008, S. 82 84, S. 82.

26 Vgl. Armin Pfahl-Traughber, a.a.O. (Fn. 21), S. 65.

27 Vgl. Fabian Virchow, Dimensionen der Demonstrationspolitik der extremen Rechten in Deutschland, in: Andreas Klärner / Michael Kohlstruck (Hrsg.), Moderner Rechtsextremismus in Deutschland, Hamburg 2006, S. 68 - 101, S. 69. 
halb der Szene zur Demonstrationsteilnahme zu bewegen, um der Öffentlichkeit eine Partei zu präsentieren, die nah am Volk steht. ${ }^{28}$

Aus diesen Überlegungen heraus veränderten die Funktionäre auch das Spektrum ihrer Slogans: Ging man Ende der 1990er Jahre noch unter dem in die NS-Zeit wirkenden Motto „Unsere Großväter waren keine Verbrecher“29 auf die Straße, wurde im letzten Jahrzehnt vermehrt an gesellschaftliche Problemlagen angeknüpft; so haben in jüngster Zeit die NPD-Aktivisten gegen die „unsoziale“ Hartz-IV-Politik und die Macht der Banken gewettert. ${ }^{30}$ Die Themen des Bundestagswahlprogramms waren außerdem Arbeit, Rente, bezahlbare Wohnungen und Energieversorgung ${ }^{31}$; auch in der Globalisierung und dem Kapitalismus begannen die Strategen der NPD ihren neuen Feind auszumachen ${ }^{32}$ - überwiegend Themen und Einstellungen, die eigentlich der politisch Linken zugeordnet werden. Bei der NPD sind jedoch selbst diese subtil mit Aversionen gegenüber Ausländern aufgeladen.

In diesem Zuge entdeckten die Rechtsextremen die Anschlussfähigkeit sozialistischer Konzepte und begannen diese für ihre öffentliche Wirkung fruchtbar zu machen. Laut Jürgen Gansel bestünde gar Wählerpotenzial bei den Traditionsbeständen der Linken. Durch die Anpassung der Linken an das System und ihre Gleichgültigkeit gegenüber den „Globalisierungsopfern“ sei eine „Vertretungslücke“ entstanden, die von der NPD gefüllt werden könne. ${ }^{33}$ Gansel bezeichnet sich selbst als rechtsextrem in der Rassenfrage, aber linksextrem beim Anti-Kapitalismus und möchte mit der NPD eine „raumorientierte Volkswirtschaft “34 schaffen. Allgemein versteht sich die Partei in jenen Aspekten als rechts, die das „Lebensrecht des Deutschen Volkes“ sowie seine Kultur und Sprache betreffen. Gleichzeitig agiert sie in ihrem Selbstverständnis links, wenn es um die soziale Frage, die Globalisierung und den Anti-Kapitalismus geht. ${ }^{35}$ Jene Ausrichtung zielt vor allem auf Personen aus sozial schwachen Gegenden oder Positionen ab.

Daneben waren stets junge Menschen im Fokus. Mittels einer von Jan Raabe als „EventAktionismus“ bezeichneten Strategie der Konzerte und Sommerfeste sollten diese an die Partei gebunden werden. Hierbei wurde auch auf Jugendliche außerhalb der Szene gezielt, um sie für politischen Aktivismus zu begeistern. ${ }^{36}$ Besonders wirkungsvoll erschien der NPD dafür die Musik, die als Einstiegsfaktor und wichtiges Medium zur Verbreitung von rechter Ideologie bekannt ist. Die infamen „Schulhof-CDs“, kostenlos verteilte Tonträger mit stark nationalistischen und teils rassistischen Inhalten, dienten nicht nur als Köder für junge Menschen - sie waren ein gelungenes PR-Instrument, um wiederholt im Lichte der Öffentlichkeit stehen zu können.

28 Vgl. Armin Pfahl-Traughber, a.a.O. (Fn. 21), S. 56.

29 Motto der Demonstration gegen die „Wehrmachtsausstellung" 1997 in München, siehe hierzu auch Fabian Virchow, Gegen den Zivilismus. Internationale Beziehungen und Militär in den politischen Konzeptionen der extremen Rechten, Wiesbaden 2006, S. 430.

30 Vgl. NPD, Bundestagswahlprogramm, Berlin 2013, http://npd.de/inhalte/daten/dateiablage/ br_2013_wahlprogramm_netz.pdf (Abruf am 16. Mai 2014), S. 9, S. 21.

31 Vgl. ebenda.

32 Vgl. NPD, Wo steht die NPD, http://www.npd-niedersachsen.de/index.php/menue/55/thema/1647/Wo_steht_die_NPD.html (Abruf am 17. Juni 2013).

33 Vgl. Armin Pfabl-Traughber, a.a.O. (Fn. 21), S. 40.

34 Jürgen Gansel, a.a.O. (Fn. 16).

35 Vgl. NPD, a.a.O. (Fn. 30).

36 Vgl. Jan Raabe, Wie versucht die NPD Jugendliche anzusprechen, in: Fabian Virchow / Christian Dornbusch (Hrsg.), a.a.O. (Fn. 25), S. $85-87$. 
Derlei Berichterstattungen spielen der Partei in die Hände, und ihre Akteure nutzen Gelegenheiten zur öffentlichen Diskussion ausgiebig. Der Ausdruck „national befreite Zone“ war eines der bekanntesten Beispiele mit einschlägiger Wirkkraft. NPD-Funktionäre ließen ihn reaktiv in die Öffentlichkeitsstrategie einfließen, weil er in den Medien und der Öffentlichkeit weitläufig diskutiert wurde. So wurde er strategisch innerhalb der Partei wiederbelebt. In der Konzeptschrift für die „national befreite Zone“ - eine Idee der JN und des nationaldemokratischen Hochschulbundes (NHB) - werden die Zonen als kommunikative und finanzielle Freiräume ausgelegt. ${ }^{37}$ Dem Ansatz zufolge sollen Gebäude erworben werden, in denen ungestört Konzerte, Fortbildungen, Tagungen etc. abgehalten werden können. Die mediale Wirkung des Begriffs ist klar: Er verleiht der Öffentlichkeit eine Vorstellung, die dem Wirkungsfeld der NPD weitaus mehr Einfluss zuschreibt, als in Wirklichkeit vorhanden ist. 38

Nach einer ähnlich öffentlichkeitswirksamen Strategie verfahren ihre Politiker im Parlament. Hier werden Anträge und Kleine Anfragen in großer Zahl gestellt: Unter den Parlamentsdokumenten des Sächsischen Landtages finden sich bis Mitte Mai 5.370 Kleine Anfragen der NPD-Fraktion an die Landesregierung - innerhalb von nur zwei Wahlperioden. 39

Hinsichtlich ihrer Wirkung in der Öffentlichkeit hat sich die Partei nunmehr auf ein weiteres Konzept eingeschworen: die Selbstdarstellung als Opfer und Sündenbock. Ein Antrag auf die eigene Prüfung auf Verfassungskonformität beim Bundesverfassungsgericht schuf solch eine medienwirksame Debatte. Aber auch die Spekulationen um die Rolle des Verfassungsschutzes im Falle des NSU spielten hierbei eine Rolle: Die Verschwörungstheorien, in denen gemutmaßt wird, dass der Verfassungsschutz aktiv die Organisation unterstützte, stammen eigentlich aus dem linken Spektrum. Akteure der NPD haben dies jedoch geschickt in ihre Strategie integriert und propagieren den gleichen Standpunkt wie ihre politischen Gegner. ${ }^{40}$ Sie interpretieren die „Verschwörung“ aber in der Weise, dass dies ein Plan gewesen sei, um die rechte Bewegung in Deutschland zu schwächen.

In jüngster Zeit suchten die Aktivisten nach neuen Wegen zur Anschlussfähigkeit an die Gesellschaft; sie fanden diese in Protesten gegen Asylbewerberheime. Die ablehnende Haltung der Bürger gegenüber den Asylsuchenden konnte in Gemeinden wie Hellersdorf und Schneeberg genutzt werden. Dies ist Teil der Gesamtstrategie, denn der „Kampf um die Straße“ sollte die notwendige Vorarbeit für den „Kampf um die Parlamente“ leisten. Die Öffentlichkeitsarbeit hatte aber vor allem das Ziel, Personen aus der „Mitte der Gesellschaft" anzusprechen, denn hier sind schließlich die meisten Wählerstimmen zu gewinnen. Für die Partei heißt dies allerdings, dass sie sich - zumindest nach außen hin - gesellschaftlich vertretbar präsentieren muss.

37 Vgl. Uta Döring, Was ist eine national befreite Zone, in: Fabian Virchow / Christian Dornbusch (Hrsg.), a.a.O. (Fn. 25), S. $94-96$, S. 94 f.

38 Vgl. ebenda, S. 96.

39 Vgl. Sächsischer Landtag, Parlamentsdokumentation, http://edas.landtag.sachsen.de/ (Abruf am 30. Mai 2014).

40 Vgl. Marc Brandstetter, Kinderfeste hinter Stacheldraht : die Entwicklung der NPD in Mecklenburg-Vorpommern nach der Landtagswahl 2011, in: ZParl, 44. Jg. (2013), H. 1, S. 146 - 157, S. 151 . 


\subsection{Wo die Wählerstimmen sind: Eine Annäherung an die politische Mitte}

Dem „Kampf um die Parlamente“ liegt de facto der Gedanke einer Annäherung an die Gesellschaft zugrunde. Unter Udo Voigt wurde dieses Mittel erprobt und internalisiert; er hatte schnell erkannt, dass das rechte Wählerpotenzial nie ausreichen würde, um die angestrebte politische Wirksamkeit zu erlangen. Holger Apfel entwickelte diese Strategie zur „seriösen Radikalität"', um die Partei weg vom rechten Rand in die Mitte der Gesellschaft zu befördern. „Aus der Mitte des Volkes“ stellt sich auch die NPD-Fraktion Sachsens den Zuschauern eines Werbevideos vor. ${ }^{41}$

Um gesellschaftsnah zu erscheinen, sollten die offen rechtsextremen und neonazistischen Einstellungen innerhalb der NPD verschleiert werden. Darum setzt die Partei im Zuge ihrer Annäherungsstrategie beispielsweise auf das Ethnopluralismuskonzept der Neuen Rechten $^{42}$ : Im Parteiprogramm werden dementsprechend rassistische Forderungen als die „Reinerhaltung“ des Volkes im Sinne eines „Recht[s] auf Selbstbestimmung und Wahrung kultureller und nationaler Identität “43 umschrieben. Der Anschein der Neonazi-Partei sollte so nach außen hin verschwinden, ohne der rechtsextremen Klientel eine komplette Absage zu erteilen. Im aktuellen Wahlprogramm wird zu Beginn weiterhin ein scheinbares Bekenntnis an den demokratischen Verfassungsstaat formuliert, das allerdings auch als Kampf für einen NS-Staat ausgelegt werden kann: „Die NPD hält an der Ordnung des Nationalstaates fest, in dessen Rahmen unser Volk seine Werte pflegt. “44 Dabei kann spekuliert werden, welche Ordnung und Werte gemeint sind. Extreme Standpunkte sollen allerdings strategisch abgewiegelt werden: So wird im parteiinternen Handbuch empfohlen, Ausländerfeindlichkeit als ,Inländerfreundlichkeit‘ zu bezeichnen oder in Diskussionen Bezüge auf Adolf Hitler abzuschmettern und das Gespräch auf andere politische Themen umzulenken. ${ }^{45}$

Doch nicht nur das Verschleiern von extremistischen Einstellungen stand im Fokus der strategischen Neuorientierung: Zusammen mit dem positiven Wirken in die Öffentlichkeit hinein werden vermehrt Themen der politischen Mitte angesprochen, um Bürgernähe zu demonstrieren. Dieses Spektrum reicht von sozialen und bildungspolitischen Fragen bis hin zum Umwelt- und Tierschutz, zum Beispiel die Ablehnung von Fracking und Tierversuchen. ${ }^{46}$ Allerdings sind politische Standpunkte gegen Ausländer nicht aus der NPD-Programmatik verschwunden, sondern wurden lediglich von den genannten Themen umschlossen und damit weniger sichtbar. Im Wahlprogramm 2013 findet sich die Agitation gegen Ausländer erst gegen Ende des Papiers - auf Seite 41 - und ist mit „Vorrang für Inländer “ 47 betitelt. Die fremdenfeindlichen Einstellungen bleiben also bestehen; gesellschaftliche Probleme, die der deutschen Sozialpolitik geschuldet seien, sollen jener Thema-

41 Homepage der NPD-Fraktion im Sächsischen Landtag, http://www.npd-fraktion-sachsen.de/ fraktion/ (Abruf am 19. Juli 2013).

42 Vgl. Uwe Backes, Die Entzauberung der Extremisten? Erfolgsbedingungen der NPD im internationalen Vergleich, Sankt Augustin / Berlin 2008, S. 55.

43 NPD, Parteiprogramm „Arbeit. Familie. Vaterland“, Berlin 2010, http://npd.de/inhalte/daten/ dateiablage/br_parteiprogramm_a4.pdf (Abruf am 16. Mai 2014), S. 13.

44 NPD, a.a.O. (Fn. 30), S. 8.

45 Vgl. NPD-Parteivorstand / Amt für Öffentlichkeitsarbeit, Argumente für Kandidaten und Funktionsträger, Berlin 2006, S. 6, S. 34.

46 Vgl. NPD, a.a.O. (Fn. 30), S. 29, S. 53.

47 Ebenda, S. 41. 
tik und vor allem der Verherrlichung des Nationalsozialismus jedoch vorgeschoben werden - Hartz IV, Globalisierung und „Verausländerung“ sind die Themen, die diskutiert werden sollen. ${ }^{48}$

Die Annäherung an die Gesellschaft soll nicht nur durch eine angepasste Programmatik gefördert werden, sondern auch durch alltagspolitische Verankerung und stete Präsenz auf kommunaler Ebene. ${ }^{49}$ Ist dort eine Vertrauensbasis erreicht, kann der Einzug in die Landtage und schließlich auch in den Bundestag gelingen ${ }^{50}$, denn wählt man kommunal NPD, so würde man dies auch auf anderen Ebenen $\operatorname{tun}^{51}$ - so lautet zumindest die Strategie.

Besondere Aufmerksamkeit für die Verwirklichung dieser Ziele schenkte die NPD-Parteiführung den ostdeutschen Bundesländern. Die politischen, kulturellen und ökonomischen Strukturen erkannten die Funktionäre schnell als günstigen Ansatzpunkt: Mit einer geringeren Demokratiezufriedenheit und schwächeren zivilgesellschaftlichen Strukturen als im Westen erschienen die Bundesländer im Osten für eine Anknüpfung geeigneter. Zudem gab es dort bereits eine breite rechtsextreme Szene. ${ }^{52}$

Die Arbeit in Dörfern und Gemeinden soll das Bild einer Partei liefern, die nah am Menschen ist. So wurden in strukturschwächeren Regionen Bürgerbüros etabliert, in denen konkrete Unterstützung wie beispielsweise Bewerbungshilfen angeboten werden. Insbesondere Pastörs wird ein großer Anteil am Erfolg der Partei in Mecklenburg-Vorpommern zugeschrieben. Programmatisch setzt die NPD gleichzeitig auf die Nationalisierung sozialer Fragen, um sich mit der Mischung aus Ethnonationalismus und Anti-Kapitalismus Menschen in schwieriger Lage anzunähern. ${ }^{53}$ Hier bedienen sich die Kader populistischer Methoden und verkünden, dass heute die „Gewinner [...] die Banken und die Finanzindustrie sind, während Otto Normalverbraucher auf der Strecke bleibt" ${ }^{\text {"54 }}$.

Bei Gemeinderatswahlen versucht die NPD seither auf Personen zu setzen, die den Einwohnern persönlich bekannt sind. ${ }^{55}$ Möglichst greifbare Politik zu präsentieren ist das Ziel. Mit den Wahlerfolgen in Sachsen und Mecklenburg-Vorpommern galt das Konzept als bewährt und sollte auf andere Bundesländer übertragen werden. So versuchte die NPD in Bayern, mit Flugblattaktionen und Pressemitteilungen die Nähe zu Familien, Jugendlichen und Landwirten zu gewinnen. Andreas Speit sieht darin eine Art Blut- und Bodenideologie, die in einer ökologischen Thematik zum Tragen kommt. ${ }^{56}$

48 Vgl. NPD-Parteivorstand / Amt für Öffentlichkeitsarbeit, a.a.O. (Fn. 45).

49 Vgl. Christian Dornbusch / Fabian Virchow, Hat die NPD eine Strategie zur Machtergreifung, in: dies. (Hrsg.), a.a.O. (Fn. 25), S. 70 - 73, S. 72.

$50 \mathrm{Vgl}$. Friedemann Bringt / Christian Dornbusch, Was macht die NPD in den kommunalen Parlamenten, in: Fabian Virchow / Christian Dornbusch (Hrsg.), a.a.O. (Fn. 25), S. 127 - 129, S. 128.

51 Vgl. Fabian Virchow, a.a.O. (Fn. 27), S. 71.

52 Der Rechtsextremismus ist jedoch kein ostdeutsches Problem: Viele Funktionäre wurden aus dem Westen für diese Gegenden rekrutiert. Man wählte dieses Gebiet gezielt als Angriffspunkt, weil es sich strukturell dafür anbot.

53 Vgl. Armin Pfahl-Traughber, a.a.O. (Fn. 21), S. 40.

54 Holger Apfel, Schäubles Liste, in: „Klartext 33“, NPD-Fraktionszeitung im Sächsischen Landtag, S. 1.

55 Vgl. Hubertus Buchstein / Gudrun Heinrich, Rechtsextremismus in Ostdeutschland - Demokratie und Rechtsextremismus im ländlichen Raum, Schwalbach 2010, S. 433.

56 Vgl. Ulrich Hagmann, Braune Ökos, Rechtsextreme unterwandern die Biobewegung, http:// www.br.de/fernsehen/das-erste/sendungen/report-muenchen/dossiers-und-mehr/report-muenchen-rechtsextreme100.html (Abruf am 26. August 2013). 
Neben jungen, radikalen Anhängern hat sich die NPD nunmehr auf eine andere Zielgruppe konzentriert: Familien, besonders mit kleinen Kindern, rückten in den Hauptfokus der Mitgliederanwerbung; Wandertage und Basare für Kinderbedarf wurden von Parteiaktivisten organisiert. In Bayern sollten mit dieser Strategie rechtskonservative CSU-Wähler erreicht werden. Auch hier wurde an vorhandene Ressentiments angeknüpft und kommunalpolitische Themen besetzt; deshalb gesellte sich die NPD beispielsweise zu Bürgerinitiativen, wie Proteste gegen die Erweiterung des Flughafens in München. ${ }^{57}$ Annett Mängel erkannte dies als Teil einer langfristig angelegten Strategie, mit der nach und nach die Verankerung in der Alltagskultur der Gesellschaft gelingen sollte. ${ }^{58}$ Diese Präsenz im öffentlichen Leben bezeichnet Marc Brandstetter treffend als „Dauerwahlkampf“59.

Schnell stieß die NPD mit ihrem strategischen Grundgerüst auf ein Problem: Weder für die Demonstrationspolitik noch für weitreichende Kommunalarbeit stand genug Personal aus den eigenen Reihen zur Verfügung. Darum war die Partei stets auf die Unterstützung von autonomen Neonazi-Organisationen - insbesondere den „Freien Kameradschaften“ angewiesen.

\subsection{Die NPD als Speerspitze des „Nationalen Widerstandes“}

Der politische Aufstieg der NPD in den 2000er Jahren war vor allem aufgrund des Zutuns von Personen aus der extrem rechten Szene möglich. Als in den 1990er Jahren zahlreiche Verbote von Neonazi-Gruppierungen erlassen wurden, wechselten viele dieser Aktivisten zur NPD. Armin Pfahl-Traughber zählt diese zur nationalsozialistischen Strömung in der Partei. ${ }^{60}$ Ihre direkte Anhängerschaft war selbst damals zu klein, um in den Hauptaktionsgegenden Sachsen und Mecklenburg-Vorpommern agieren zu können - obwohl vor allem in Sachsen Personal aus dem gesamten Bundesgebiet gebündelt wurde. Deshalb hatte Voigt den Versuch gestartet, die Spaltung im „nationalen Lager“ zu überwinden und die Symbiose militanter Neonazis mit traditionellen Parteirechten zu vollziehen. ${ }^{61}$

Diese Ende 2004 als „Kampf um den organisierten Willen“ geschaffene Säule sollte die rechtsextreme Bewegung unter der Führung der NPD einen. Praktisch ging es vor allem um die Kooperation mit den „Freien Kameradschaften“. Diese sind Netzwerke von Neonazis, die personelle und ideologische Gemeinsamkeiten mit extremistischen Parteien aufweisen, sich aber im strategischen und taktischen Vorgehen von diesen unterscheiden. ${ }^{62}$ Bevor Voigt Parteivorsitzender wurde, bestand eine innerparteiliche Abgrenzungspolitik zum Neonazi-Lager ${ }^{63}$, für die der Parteivorstand 2004 keinen Anlass mehr sah. Dies brachte das erste Parteiverbotsverfahren (2000 bis 2003) mit sich: Zunächst distanzierte sich der Füh-

57 Vgl. Robert Andreasch / Andreas Speit, Mit Schwung in den Westen? Wie sich die NPD eine Zukunft in Bayern vorstellt, Bundeszentrale für politische Bildung, 17. Juni 2008, http://www.bpb. de/themen/B0NDCN,2,0,Mit_Schwung_in_den_Westen.html (Abruf am 15. Mai 2013).

$58 \mathrm{Vgl}$. Annett Mängel, Ganz normal rechts, in: Blätter für deutsche und internationale Politik 11/2006, S. 1295 - 1298, S. 1295.

59 Marc Brandstetter, a.a.O. (Fn. 40), S. 153.

60 Vgl. Armin Pfahl-Traughber, a.a.O. (Fn. 21), S. 28.

61 Vgl. Toralf Staudt, Moderne Nazis, Bonn 2006, S. 25.

62 Vgl. Fabian Virchow, a.a.O. (Fn. 29).

63 Vgl. Marc Brandstetter, Die NPD im 21. Jahrhundert, Marburg 2006, S. 86. 
rungskader zunehmend von extremen Rechten; das Scheitern des Verbots verlieh der NPD jedoch Aufschwung und spielte ihr in die Hand - denn zeitgleich war sie vermehrt auf die Freien Kräfte angewiesen. Die neu gewonnene Legitimität mündete in einer nahezu uneingeschränkten Öffnung für „Freie Kameradschaften“, Neonazi-Größen und rechtsextreme Musiker. Insgesamt stärkte das gescheiterte Verbotsverfahren die Partei in ihrem Selbstverständnis und wurde in rechten Kreisen als ein Aufbruchsignal gesehen. ${ }^{64}$

\section{Umsetzbarkeit der Strategie}

\subsection{Die pseudowissenschaftliche Intellektualisierung}

Trotz intensiver Bemühungen um eine Intellektualisierung ist dieser Teil der Strategie nicht von Erfolg gekrönt, obwohl dumpfe rechte Parolen seltener als in der Vergangenheit zu vernehmen sind. Vielmehr legen Parteimitglieder Wert auf Preisgabe ihres Wissens aus Geschichtsbüchern; so deutet etwa Gansel die Geschichte Preußens zum 300. Geburtstag von Friedrich dem Großen. ${ }^{65}$ Publizisten der Partei interpretieren wissenschaftliche Erkenntnisse zuweilen auf Verteidigung und Legitimation ihrer pseudowissenschaftlichen Positionen hin; Richter beruft sich beispielsweise auf Soziologen und Bevölkerungswissenschaftler, dabei deutet er Samuel Huntingtons Idee des Kampfs der Kulturen um. ${ }^{66}$ Aufgrund jener Versuche versuchen Waldhoff und Möllers im Verbotsantrag das „Bildungswerk für Heimat und nationale Identität e.V.“ als gelungenen Prototyp für die Vermittlung von intellektuellem Rechtsextremismus darzustellen. ${ }^{67}$ In der Tat sind ihre NPD-nahen Magazine wie hier \& jetzt qualitativ höherwertig als andere Schriften der Rechten, während die Deutsche Stimme (DS) - unter der Leitung von Mattias Faust - eher zu einem rechten Boulevardblatt mutierte, so dass es über die Parteigrenzen hinaus nahezu unbeachtet bleibt. Haben Gansel und Richter vor einigen Jahren noch versucht, mit ihren Beiträgen richtungsweisende ideologische und strategische Fragen zu erörtern und in die Köpfe ihrer Anhänger und Kollegen zu befördern, ${ }^{68}$ wurde aus der DS ein Magazin, das sich damit rühmt, das zu sagen, „was andere sich nicht einmal zu denken trauen“69. Die rechtspopulistische „Berichterstattung“ spricht zumeist nur das rechte Spektrum selbst an, wenngleich das Niveau der öffentlichen Kommentatoren gestiegen ist. Dumpfe Parolen sind nun zumindest nicht mehr der Standard.

Institutionalisierte Intellektualisierungsversuche wie die Dresdner Schule waren bisher ein Misserfolg: Der von Gansel erdachte Think Tank blieb ein Luftschloss und hatte bis auf die Namensähnlichkeit mit der Frankfurter Schule nichts gemein. Auch die Stiftung ist bislang kaum politisch aufgefallen. Waldhoff und Möllers merken jedoch an, dass die im

64 Vgl. Bundesamt für Verfassungsschutz, Aktuelle Entwicklung der „Nationaldemokratischen Partei Deutschlands" (NPD) und ihr Verhältnis zu den Neonazis, Bundesministerium des Inneren, Köln 2004.

65 Vgl. Homepage von Jürgen Gansel, Zum 300. Geburtstag Friedrichs des Großen am 24. Januar 1712, 25. Januar 2012, http://juergen-gansel.de/index.php?s=13\&aid=12 (Abruf am 16. Mai 2014).

66 Vgl. Karl Richter, Krieg der Krippen, Krieg der Kulturen, in: hier \& jetzt, H. 6/07 , S. 4 - 9.

67 Vgl. Christoph Möllers / Christian Waldhoff, a.a.O. (Fn. 3), S. 33.

68 Vgl. Armin Pfahl-Traughber, a.a.O. (Fn. 21), S. 42 f.

69 Vgl. Homepage der Deutschen Stimme, http://ds-aktuell.de/?page_id=2331 (Abruf am 16. Mai 2014). 
Konzept geplanten Schulungen stark zugenommen haben. ${ }^{70}$ Dies ist auch als Erfolg des „Kampfs um die Köpfe“ zu sehen, da Aktivisten häufig plangemäß nur noch schwer als Rechtsextreme zu erkennen sind. Vor allem die Ideen um einen neuen Stil sind fast durchgehend angekommen: Anzüge, karierte Hemden, bunte Sommerkleidung - die NPD-Aktivisten fallen nicht mehr sofort negativ auf. Aber auch wenn die Partei ehemals militante Neonazis schulen und integrieren konnte, ist die Partei von einer intellektuellen Front weit entfernt. Die Rechtsextremen wirken nur im Vergleich zu den Parolen dreschenden Neonazis der 80er und 90er Jahre gebildeter. Sogar die Akteure der Neuen Rechten grenzen sich mehrheitlich aus diesen Gründen von der NPD ab.

Deren Konzepte wurden jedoch vielfach von Funktionären fruchtbar gemacht. Sie verschleiern ihre rassistischen Einstellungen durch Ideen und Mimikry-Strategien der NR, beispielsweise wenn sie in ihrem Parteiprogramm unterschiedliche „Rassen“ in der „Vielfalt der Völker als Träger der Kulturen“71 erkennen und „[s] ogenannte ,multikulturelle“ Gesellschaften [...] in Wirklichkeit kulturlose Gesellschaftsformen [sind], die je nach ihrer ethnokulturellen Zusammensetzung Parallelgesellschaften ausbilden, die für jedes Staatswesen zur Zerreißprobe werden“72. Allen Bemühungen zum Trotz wird die gedankliche Grundlage des völkischen Nationalismus nahezu jedem Beobachter klar. Hinter dem angepassten Auftreten der NPD stehen dasselbe Gedankengut des völkischen Nationalismus, und die Verarbeitung wissenschaftlicher Ansätze reicht für eine intellektuelle Fassade nicht aus. Auch die Adaption von Termini und Konzepten linker Theoretiker bringt kaum mehr Legitimität eines rassistischen Kerns. Mit jenen Einstellungen kann die Partei schwerlich positiv in die Gesellschaft wirken.

\section{2. Öffentlichkeitsarbeit und ihr Gegeneffekt}

Die Elemente der Öffentlichkeitsarbeit konnten nur geringen Erfolg verzeichnen. Vor allem ist das Konzept der „Demonstrationspolitik“ gescheitert, denn diese Aktionsform konnte meist nur in Zusammenarbeit mit den so genannten Freien Kräften gelingen. Mit dem angestrebten bürgerlichen Erscheinungsbild der Partei war dies nicht vereinbar: Die Präsenz von militanten Neonazis trübte die Strategie der Seriosität, aber das angepasste Lager der NPD war zu klein für eine erfolgreiche Präsenz auf der Straße, und außerhalb der rechtsextremen Klientel war kein relevantes Potenzial zur Teilnahme an Protesten bereit. Daher wirkten die jüngsten Parteiaufmärsche kläglich: Sind zu den Glanzzeiten der Demonstrationsstrategie noch tausende auf die Straße gegangen - wie in München 1997 -, versammelt sich heute oft nur eine zweistellige Personenzahl, mit der Folge, dass der Zug von ähnlich viel Polizisten begleitet wird. Die Demonstrationen büßten so massiv an öffentlichem Aufsehen ein.

Die Aufmärsche im Jahr 2013 wurden häufig sogar zur Blamage. Dieses Jahr kann als Crescendo des Widerstands gegen die NPD gesehen werden, denn oft stellte sich ihr eine Überzahl von Gegendemonstranten in den Weg, wie in Berlin-Kreuzberg. Ähnlich boten ihnen Einwohner in anderen Teilen des Landes Paroli, sei es in Fürth, Coburg oder in kleineren Orten wie Gröbenzell, wo NPD-Aktivisten ihren Infostand aufgrund des Widerstan-

70 Vgl. Christoph Möllers / Christian Waldhoff, a.a.O. (Fn. 3), S. 34.

71 NPD, a.a.O. (Fn. 43), S. 6.

72 Ebenda, S. 9. 
des von Kommunalpolitikern und Bürgern abbauten. Insgesamt müssen sich die Aktivisten eingestehen, dass ihre Anwesenheit von der Mitte der Gesellschaft nicht erwünscht ist. Das Scheitern der Demonstrationsstrategie ist ein Problem für die Partei: Sie einte die häufig zerstrittene rechte Szene in der Vergangenheit. Damit verlor die NPD ein wichtiges Mittel für ihre öffentliche Wirksamkeit.

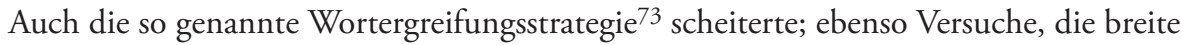
Masse der Jugend mit Konzerten und Sommerfesten zu erreichen. Die einzige Aktionsform, der im letzten Jahr ein nennenswerter Erfolg zugeschrieben werden kann, ist die Instrumentalisierung der breiten Ablehnung von Asylbewerbern in einigen Regionen Deutschlands. Dabei traten aber die Funktionäre der Partei zumeist verdeckt auf.

Während die NPD zu Beginn des Jahrtausends noch für ein fremdenfeindliches Klima in der Bundesrepublik sorgen konnte ${ }^{74}$, wird auf sie im Jahr 2013 in den meisten Teilen Deutschlands nur noch reagiert, wenn eine skandalöse Aussage, ein anstößiges Wahlplakat oder die Nachricht über eine belanglose Ansammlung ihrer wenigen Mitglieder in den Medien auftaucht. In jüngster Zeit kommt das Bild einer in sich zerstrittenen Partei immer weiter zum Vorschein. Nicht nur der Parteivorsitzende Apfel stolperte über Vorwürfe von sexuellem Fehlverhalten, auch der NPD-Generalsekretär Peter Marx trat aufgrund ähnlicher Vorwürfe zurück.

Erfolgreich können die Aktivisten nur dort erscheinen, wo sie sich verdeckt unter Gruppen von Personen mischen, an deren rechtslastigem Gedankengut sie anknüpfen können. Der Parteiname gilt weiterhin als stark stigmatisiert, so dass die breite Mitte der Gesellschaft von der NPD kaum erreicht wird. In der Vergangenheit konnte die Strategie in manchen Gegenden zwar beachtliche Teilerfolge erzielen, der Rückhalt schwindet jedoch auch hier und ihre Ausweitung ist nahezu unmöglich.

\subsection{Der verlorene „Kampf um die Parlamente“}

Die Annäherung an die Mitte der Gesellschaft kann als wichtigster und daher auch am stärksten forcierter Teil der Strategie betrachtet werden. Durch Kommunalarbeit erlangte die NPD in der Tat eine neue Position; vor allem in den dünn besiedelten Gebieten Mecklenburg-Vorpommerns ist sie nach wie vor relativ stark. ${ }^{75} \mathrm{Im}$ Verbotsantrag wird daher formuliert, dass die Partei in ihrem Ziel, die demokratische Ordnung im ganzen Land abzuschaffen, auf lokaler Ebene „bereits Beeinträchtigungen dieser Ordnung erreicht hat"76. Tatsächlich birgt die Strategie der lokalen Verankerungen in einigen Gegenden Gefahr. Insbesondere wegen ihres Wirkens in Dörfern und im Schweriner Parlament ist es verständlich, dass sich so mancher Bürger oder Politiker in Mecklenburg-Vorpommern ein Verbot wünscht. De facto hat die NPD jedoch keinen solchen Einfluss, dass die demokratische Ordnung beeinträchtigt würde. In Untersuchungen und Berichten wurden Ausnahmen fälschlicherweise zu Erfolgsbeispielen erklärt, etwa der Fall der Gemeinde Reinhardts-

73 Auf öffentlichen Veranstaltungen demokratischer Parteien sollten Parteimitglieder aus dem Publikum heraus das „Wort ergreifen“ und die Redner in Diskussionen verwickeln, um diese möglichst unfähig erscheinen zu lassen und Alternativen aufbieten zu können.

$74 \mathrm{Vgl}$. Eckhard Jesse, a.a.O. (Fn. 4).

75 Vgl. Marc Brandstetter, a.a.O. (Fn. 40), S. 149.

76 Christoph Möllers / Christian Waldhoff, a.a.O. (Fn. 3), S. 8. 
dorf-Schöna, wo die Partei bei der Wahl 22,0 Prozent der Stimmen erhielt. Aber schon die Bürgermeisterwahl im März 2013 zeigte ein ganz anderes Bild: Der NPD-Kandidat konnte lediglich drei Stimmen verbuchen. ${ }^{77}$

Insgesamt deutet vieles auf einen Einbruch in ihren Erfolgsgegenden hin: Ihre lokale Schwerpunktsetzung in Mecklenburg-Vorpommern führte zunächst zu einer kontinuierlichen Zunahme an Wählerstimmen. Bei der Landtagswahl 2002 erzielte die NPD lediglich 0,8 Prozent der Zweitstimmen, bei der Bundestagswahl 2005 schon 3,5 Prozent. Nach der Etablierung einiger Bürgerbüros und mit Hilfe der Freien Kräfte zog sie im Herbst 2006 dann mit 7,3 Prozent in den Landtag ein. Dass 2011 der Stimmenanteil auf 6,0 Prozent fiel, dies ist jedoch nicht nur auf die damals schon beginnenden Zerwürfnisse mit einigen „Freien Kameradschaften“ zurückzuführen: Die Partei verlor gleichzeitig an Attraktivität und Ansehen. Es scheint, als ob die Wahlerfolge der NPD eher geschadet haben. Ihre Instrumentalisierung des Parlamentes zeigte, dass sie Politik nicht als programmatischen Diskurs versteht, sondern ihrer geschlossenen Weltanschauung starr anhaftet; damit können sich die Bürger nicht identifizieren. Die letzte Emnid-Umfrage vom 14. September 2013 sah die NPD bei gerade noch 5,0 Prozent; eine Erhebung vom März 2014 ergab lediglich 1,1 Prozent an Wählerstimmen. ${ }^{78}$ Die Auszählung der Ergebnisse zur Wahl des Europäischen Parlaments vom Mai 2014 brachte 3,0 Prozent. ${ }^{79}$

Die Partei ist strukturell nicht in der Lage, ihre lokalen Konzentrationspunkte weiter auszubauen - es mangelt an Personal. Die aktive Szene um die Führungsriege in Mecklenburg-Vorpommern schätzt Marc Brandstetter auf rund 400 Personen $^{80}$ - zu wenig, um in einem ganzen Bundesland Dauerwahlkampf zu führen.

Der Rückgang ist auch einem finanziellen Engpass geschuldet, da die Bundespartei aufgrund gefälschter Spendenquittungen in Rechenschaftsberichten Schuldenberge anhäufte. Auch von dem Schaden, den der Schatzmeister Erwin Kemna verursachte, als er Parteigelder veruntreute, scheint sich die Parteikasse noch nicht erholt zu haben. Die Lage verschlimmerte sich noch mit dem Tod Jürgen Riegers und dem Wegfall seines finanziellen Rückhalts. Die Partei musste sogar Mitarbeiter entlassen und hatte des Öfteren Finanzierungsschwierigkeiten für die Wahlkampagnen. So erscheint selbst die Aufrechterhaltung der lokalen Arbeit fraglich. Die Autoren des Verbotsantrages erkennen an, dass dieses Problem zu keiner „erkennbaren Einbuße an politischer Wirksamkeit geführt" ${ }^{\text {"81 }}$ habe.

Doch selbst mit funktionierender struktureller und finanzieller Versorgung wäre eine Annäherung an die Mitte der Gesellschaft nur in wenigen Teilen Deutschlands überhaupt möglich - dies zeigte sich nicht zuletzt bei der Bundestagswahl: Mit 1,3 Prozent der Wählerstimmen entschied sich zwar kein unerheblicher Anteil der Deutschen für die NPD, eine tatsächliche politische Wirksamkeit ist der Partei damit jedoch versagt - und damit ist auch der Punkt hinfällig, den Möllers und Waldhoff in ihrem Antrag verfolgen. Nicht annähernd

77 Vgl. Amtsblatt Bad Schandau, Nummer 5, 8. März 2013, http://www.bad-schandau.de/stadt/ amtsblatt/bad_schandau_10-13.pdf (Abruf am 14. Mai 2013).

78 Vgl. Wilko Zicht / Matthias Cantow, Umfragen Mecklenburg-Vorpommern, http://www.wahlrecht.de/umfragen/landtage/mecklenburg-vorpommern.htm (Abruf am 24. März 2014).

79 Vgl. Die Landeswahlleiterin Mecklenburg-Vorpommern, Europawahl in Mecklenburg-Vorpommern am 25. Mai 2014, http://service.mvnet.de/wahlen/2014_eu_kom/2014_eu_kom/htm/E_ Proz.htm (Abruf am 29. Mai 2014).

80 Vgl. Marc Brandstetter, a.a.O. (Fn. 40), S. 153.

81 Vgl. Christoph Möllers / Christian Waldhoff, a.a.O. (Fn. 3), S. 30. 
kann die NPD ihr Ziel erreichen, die bestehende Ordnung im gesamten Bundesgebiet abzuschaffen.

Die Rhetorik der Parteimitglieder macht es geradezu unmöglich, dass die NPD flächenmäßig an Macht gewinnt: Obwohl die meisten ihrer Mitglieder, die in der Öffentlichkeit stehen, die gemäßigte Programmatik internalisiert haben und generell nach dem strategischen Konzept der Abwiegelung verfahren, können sie ihre extremistische Ausrichtung nicht verhüllen. Ausreißer wie die extremen Hetzen von Pastörs sind bekannt. Außerdem findet trotz der Übernahme des Ethnopluralismuskonzeptes die Programmatik der Partei in der breiten Gesellschaft keinen Anklang. Trotz der Verschleierung wird der extremistische Kern dieses Konzepts schnell klar, weil fundamentale Elemente der Freiheitlich demokratischen Grundordnung negiert werden - allen voran das menschliche Gleichheitsgebot. Damit offenbart sich eine totalitäre und demokratiefeindliche Ideologie, der ein Antiindividualismus, Antiliberalismus und Antisemitismus innewohnt. Allen „positiven“ Formulierungen zum Trotz: Die politische Mitte schreckt verständlicherweise ab, dass die „Reinheit des Volkes" angestrebt wird. So hilft auch kein scheinbar gemäßigtes Programm mit sozialpolitischen Inhalten, wenn gleichzeitig von einem „Schutz vor Überfremdung mittels Rückkehrpflicht statt Bleiberecht“82 und „Integration ist Völkermord“83 gesprochen wird.

Die gesellschaftliche Ablehnung der NPD ist allein daran zu sehen, dass ihr Engagement in den meisten Regionen auf Widerstand stößt: Der Erwerb von Immobilien scheiterte häufig, und nach wie vor lehnen Hotels und Gaststätten Aufträge und Buchungen von NPDFunktionären ab. ${ }^{84}$ Zum Beispiel im Coburger Umland wurden Veranstaltungen durch massiven Druck in der Bevölkerung abgesagt. Selbst für den Bundesparteitag 2013 war es schwer, einen Veranstaltungsort zu finden. Als dieser dann in Weinheim stattfinden konnte, erschienen nicht einmal 200 Personen. Die stornierten Reservierungen und Gegeninitiativen aus der Gesellschaft zeugten von einer Schmach erster Güte. Darum verwundert es kaum, dass auch die Landtagswahl 2013 in Niedersachsen bereits ein Schlag für die NPD war: 0,8 Prozent der Wählerstimmen reichten nicht einmal für die Wahlkampfkostenerstattung.

Auch in ihrer Hochburg Sachsen steht sie knapp davor, gesellschaftlich besiegt zu werden. Im Landtag rief die Wahl der NPD einen parteiübergreifenden Konsens gegen die Rechtsextremen hervor, und ihr Niedergang von 9,2 auf 5,6 Prozent bei den Landtagswahlen 2009 zeigte den Grad der Ernüchterung. Die Bundestagswahl 2013 hatte der Partei in Sachsen nur noch 3,3 Prozent beschert. ${ }^{85}$ Auch bei der Wahl zum Europäischen Parlament, bei der kleinere Parteien regelmäßig besser abschneiden, erreichte sie nur noch 3,6 Prozent. ${ }^{86}$ Es ist daher gut möglich, dass sie bei den Landtagswahlen 2014 die Fünf-ProzentHürde nicht mehr überschreiten wird. Die jüngsten Umfragen bescheinigten der Partei nur noch vier (Infratest dimap) beziehungsweise ein Prozent (INSA). ${ }^{87}$

82 NPD, a.a.O. (Fn. 43), S. 5.

83 Ebenda, S. 13.

84 Vgl. Uwe Backes, a.a.O. (Fn. 42), S. 59.

85 Vgl. Statistisches Landesamt des Freistaates Sachsen, Zweitstimmenverteilung ausgewählter Wahlvorschlagsträger bei der Wahl am 22. September 2013 in den Wahlkreisen des Freistaates Sachsen, Kamenz 2013, http://www.statistik.sachsen.de/wpr_neu/pkg_s10_ergli_lw.prc_ergli_lw_ v2?p_bz_bzid=BW13 (Abruf am 7. Oktober 2013).

86 Vgl. dass., Statistik Europawahl 2014, http://www.statistik.sachsen.de/wpr_neu/pkg_s10_erg. prc_erg_ew?p_bz_bzid=EW14\&p_ebene=SN\&p_ort=14 (Abruf am 28. Mai 2014).

87 Vgl. Wilko Zicht / Matthias Cantow, a.a.O. (Fn. 78). 
Eine generelle Entwarnung kann jedoch nicht gegeben werden, denn die Aktivisten schaffen es immer wieder, sich medienwirksam zu platzieren. So haben sich beispielsweise Mitglieder und Führungspersonal der NPD bei der Hochwasserhilfe engagiert und auf sich aufmerksam gemacht. Allgemein sind die Rechtsextremen jedoch nur noch ein Ärgernis für die demokratischen Parteien im Parlament, die dort zuweilen Reden außerhalb demokratischen Anstands und politischen Common Sense erdulden müssen.

Wie wenig die NPD die Mitte der Gesellschaft erreicht, bewies sich auch in Bayern, wo sie sich aufgrund fehlender Unterschriften von Unterstützern nicht einmal für eine flächendeckende Teilnahme an der Bundestagswahl qualifizieren konnte. Dies geht nicht zuletzt auch auf die Mordserie des NSU zurück, in deren Folge die Bürger erneut für Rechtsextremismus sensibilisiert wurden. Danach agierte die NPD etwas zurückhaltender - beispielsweise im sächsischen Parlament. Reden und Anträge fielen entschärfter aus und eine Abgrenzungsstrategie, die Verschwörungstheorien über die Zusammenarbeit des Verfassungsschutzes mit dem NSU sowie die Opferrolle der Partei kommunizierten, wurde lanciert. So postete Gansel auf seiner Facebook-Seite Kommentare zu Erkenntnissen im NSU-Ermittlungsverfahren: „NSU“-Trio offenbar komplett staatlich gesteuert!“. Wie ernst die Parteiführung die Stimmung gegen Rechtsextremismus in der Gesellschaft nahm, zeigte sich im Vorwort zum Programm der Bundestagswahl 2013: „[... ] bei der Bundestagswahl 2013, da sollten Sie dann die NPD wählen. Es sieht ja keiner. "88

Den Wählern können diese Kampagnen jedoch kaum etwas vormachen, denn das wahre Gesicht der NPD-Ideologie zeigt sich ihnen unentwegt. Dies übernimmt beispielsweise die Instrumentalisierung der Parlamente als Propaganda: Das Tragen von - im Parlament verbotener - Thor-Steinar-Kleidung oder das demonstrative Verlassen des Parlamentsplenums in Schwerin kurz vor einer Schweigeminute für die Opfer des NSU zeigt von rechtsstaatlichen Regeln und der Ablehnung jedweder inhaltlicher Programmatik von Politik. Folge ist die Stigmatisierung der NPD. Auch wenn sie vereinzelt Flugblätter unter die Leute bringen kann, stoßen die meisten Initiativen auf wenig Interesse bei den Bürgern. Basare und Familiennachmittage ziehen meist nur noch Anhänger aus der rechten Szene an. Und selbst wenn Presseberichte eine Annäherung von Bio-Bauern an die NPD suggerieren: Auf die „Umweltschutz ist Heimatschutz ist Volksschutz-Strategie“ geht kaum ein Wähler ein.

Darum gilt: Auch wenn sie mit der Strategie der kommunalen Verankerung im letzten Jahrzehnt Erfolge erzielt hat, stellt die Partei keine Bedrohung mehr dar - auch nicht auf kommunaler Ebene. Die lokale Fokussierung kann sie aufgrund eines Mangels an personellen und finanziellen Ressourcen nicht weiter ausbauen, eine Verankerung in den meisten Bundesländern wäre auch mit intensivster Arbeit nicht zu schaffen. Und selbst im rechtsextremen Spektrum hat die Partei Schwierigkeiten, Unterstützung zu erfahren. Dies liegt an Dissonanzen in ihrer Strategie, die sich schon länger in Konflikten niederschlagen.

\subsection{Die stumpfe Speerspitze und das Verfehlen ihres Ziels}

Aufgrund ihres theoretischen Konzeptes war die NPD dazu gezwungen, ihre Kommunikationsstrategie doppelt zu besetzen: Hetze gegen Ausländer und den demokratischen Staat

88 NPD, a.a.O. (Fn. 30), S. 6. 
musste der rechtsextremen Klientel weiterhin geboten werden. Innerparteilich mag die Übermittlung der extremistischen Positionen funktionieren, jedoch werden damit gemäßigte Wähler verschreckt, während für den Großteil der „Freien Kameradschaften“ die NPD seit langem eine „demokratische Systempartei“ ist, die sich nicht offen gegen den Staat ausspreche und von V-Männern und Spitzeln unterwandert sei.

Nahm die Partei noch vor wenigen Jahren eine federführende Rolle im Rechtsextremismus ein, ist sie nunmehr sogar in jenen Kreisen marginalisiert. Dabei bewährte sich anfangs die Öffnung hin zu den „Freien Kräften“: Führende Neonazis arbeiteten Hand in Hand mit der NPD oder traten selbst in die Partei ein - die Zuarbeit aus dem extrem rechten Lager war für sie essenziell. Harald Bergsdorf wies bereits 2007 darauf hin, dass die hohe Anzahl an westdeutschen Führungskräften in der sächsischen NPD eine Lücke zwischen Personalrekrutierung und Wählerstimmen offenbarte. ${ }^{89}$ Die „Freien Kameradschaften“ halfen damals noch, diese zu füllen. Doch die offene Kooperation mit den militanten Rechtsextremen konnte nicht gut gehen: Während die NPD die Bürger erreichen und damit Wahlen gewinnen möchte, stehen Neonazis gegen Demokratie und demokratische Gesellschaft. Auch resultierten die bürgerverträgliche Programmatik und die verordnete Unterlassung von Geschichtsrevisionismus nicht nur in Konflikten mit den „Freien“, sondern auch zwischen NPD-Funktionären. Im Zuge der Streitereien löste sich ein großer Teil der Neonazi-Szene schon vor geraumer Zeit von der Partei - eine Regelung bezüglich der Demonstrationspolitik im Jahre 2009 war dann der Auslöser für den großen Bruch.

Voigt formulierte damals einen Abgrenzungsbeschluss gegenüber den „Autonomen Nationalisten“ - eine radikale Strömung, die Auftreten und Agitation der Linksautonomen übernahm. Obwohl die Partei die „Speerspitze des Nationalen Widerstandes“ verkörpern wollte, war Voigt das „anarchische“ Konzept des schwarzen Blocks ein Dorn im Auge, da es seiner Meinung nach das Bild einer bürgernahen Partei trübt. ${ }^{90}$ Die Distanzierung von den autonomen Neonazis führte zum Aufruf der extremen Rechten, aus der NPD auszutreten. Dem folgten einige Mitglieder, vor allem aus den nationalsozialistischen Strömungen in Bayern, Sachsen und Sachsen-Anhalt. ${ }^{91}$ Seither war das Verhältnis zu den „Freien Kameradschaften“ oftmals schlecht, und parteiintern verhärteten sich zwei Fronten.

Währenddessen stiegen die „Kameradschaften“ zu den wichtigsten Gruppierungen in der rechtsextremen Szene auf. Die kleinen, dezentralisierten und im Vertrauten gehaltenen Gruppen hatten in Sachsen und Mecklenburg-Vorpommern zum Wahlerfolg der NPD beigetragen. Vor allem in Sachsen wird der Verlust jener Rückendeckung nur schwer zu verkraften sein. Mecklenburg-Vorpommern ist derzeit die einzige Region, in der das Zusammengehen von NPD und Neonazis noch einigermaßen reibungslos funktioniert. ${ }^{92}$ Der Hauptgrund hierfür dürfte die gute Vernetzung von Führungspersonen wie Udo Pastörs oder Stefan Köster sein.

Der angepasste Kurs geriet immer mehr unter Druck, und als Reaktion auf Apfels Strategie formierte sich im letzten Jahr eine innerparteiliche Gegenfront. Einem „Freundeskreis Udo

89 Vgl. Harald Bergsdorf, Die neue NPD. Antidemokraten im Aufwind, München 2007, S. 109.

90 Vgl. Marc Brandstetter, Die Selbstzerfleischung der NPD, in: Blätter für deutsche und internationale Politik 2/2009, S. 15 - 18, S. 16.

91 Vgl. ebenda.

92 Vgl. ders., a.a.O. (Fn. 40). 
Voigt “93 erschien es notwendig, alle Rechtsextremen wieder in der NPD zu vereinen und jene strategische Säule zu stärken. Dabei schien der Richtungsstreit zwischen bürgerlicher Annäherung und engem Verhältnis zu militanten Rechtsextremen zunächst zugunsten der Gemäßigten entschieden worden zu sein mit der Folge, dass weitere Mitglieder aus der NPD austraten. Nach den letzten offiziellen Angaben der Partei lag die Mitgliederzahl bei 5.400. ${ }^{94}$ Danach hätten in den letzten Jahren mehr als 2.000 Personen die NPD verlassen - dabei erscheint die Zahl von 5.400 noch hoch gegriffen, da der Trend in den Ländern den Verfassungsschutzberichten zufolge - negativ verläuft. ${ }^{95}$ Für die Entwicklung der Partei scheint vor allem Apfels Annäherungsstrategie verantwortlich gemacht zu werden. Ende 2013 trat er vom Parteivorsitz zurück; als Grund gab er ein Burnout-Syndrom an. Von einem Kollegen wurde ihm auf Facebook sogar der Selbstmord nahegelegt.

Die Strategie der Öffnung zu sämtlichen rechten Strömungen gestaltete sich als unvereinbar mit einer gesellschaftlichen Annäherung. Selbst Voigt, auf den diese Strategie eigentlich zurückgeht, kritisierte, dass die „radikale Seriosität“ die Partei viele Netzwerkstrukturen gekostet habe; er vertrete daher inhaltlich und personell eine andere Politik. ${ }^{96}$

Nach der Ablösung von Apfel durch Pastörs konnte von einem Richtungswechsel ausgegangen werden, da dieser für seine harten Positionen bekannt ist. Zunächst zeigten sich jedoch Parallelen zum ersten Parteiverbotsverfahren, bei dem die Parteiführung wieder auf Distanz ging. Der Parteiausschluss von einem ihrer bekanntesten neonationalsozialistischen Mitglieder ist ein Beispiel hierfür: Thomas Wulffbekannte sich offen zum Nationalsozialismus - für die NPD war dies ein Risiko; der Ausschluss schlug jedoch erneut Wellen ${ }^{97}$ und trug dazu bei, dass sich viele Mitglieder der „Freien Kameradschaften“ weiter von der NPD distanzierten und sich gegen die Partei aussprachen. Wulff selbst kommentierte, dass er „keine leichtfertige Gefährdung unserer Partei in einem laufenden Verbotsverfahren “98 erkennen könnte. Er forderte, sowohl den Sanktionen gegen als auch jedweder „weitere[n] Entnazifizierung der NPD“99, ein Ende zu setzen.

Aufgrund dieser Entwicklungen in den letzten Jahren scheint sich die extreme Rechte in der letzten Zeit vermehrt in die Nähe der Partei „Die Rechte“ zu orientieren. Damit ist der NPD ein großes Stück politischer Gefährlichkeit genommen: Ohne Wählerstimmen außerhalb der rechten Szene ist sie handlungsunfähig, und mit dem Verlust der extremen Rechten hat sie kaum noch politische Wirkungschancen.

93 Landesamt für Verfassungsschutz Sachsen, „Freundeskreise Udo Voigt“ gegründet, 6. Februar 2013, http://www.verfassungsschutz.sachsen.de/1385.htm (Abruf am 18. März 2013).

94 Vgl. Bayerisches Staatsministerium des Innern, für Bau und Verkehr, Verfassungsschutzbericht 2013, München 2014, S. 98.

95 Vgl. Ministerium des Innern des Landes Brandenburg, Verfassungsschutzbericht Brandenburg 2013, Potsdam 2014, S. 21.

96 Vgl. Udo Voigt, Begründung Absage meiner Teilnahme am Bundesparteitag 2013, http://wohindeutscherechte.de/?p=331 (Abruf am 10. März 2014).

97 Insbesondere das Freie Netz Süd und weitere Kameradschaften begehrten gegen den Ausschluss auf, vgl. hierzu http://www.freies-netz-sued.net/index.php/2014/04/09/nazis-raus-bei-angeschlagener-npd/ (Abruf am 25. April 2014)

98 Freies Netz Süd, Thomas „Steiner“ Wulff wehrt sich gegen NPD-Entnazifizierung, http://www. freies-netz-sued.net/index.php/2014/04/22/thomas-steiner-wulff-wehrt-sich-gegen-npd-entnazifizierung/ (Abruf am 25. April 2014).

99 Ebenda. 


\section{Das Verbot einer irrelevanten Partei}

Die in der NPD vertretene Weltanschauung ist ohne Frage verfassungswidrig. Sie ist leicht als menschenverachtend und rassistisch zu entlarven, steht aber nicht nur aus diesen Gründen den Werten westlicher Demokratie diametral entgegen. Auch eine aggressiv-kämpferische Haltung einzelner Mitglieder kann belegt werden. ${ }^{100}$ Dass die NPD den verfassungsrechtlichen Grundwerten und dem Fortbestand der verfassungsmäßigen Ordnung gefährlich wird, ist jedoch nicht ersichtlich. Noch weniger ist das vom Europäischen Gerichtshof für Menschenrechte für ein Verbot geforderte dringende soziale Bedürfnis vorhanden, die Partei aufzulösen: Der Frieden in der Gemeinschaft und die Demokratie in Deutschland sind von ihr nicht bedroht. Die Umsetzung einer parteipolitischen Strategie hängt von finanziellen, personellen und infrastrukturellen Ressourcen ab, über die die NPD nicht mehr hinreichend verfügt; deshalb ist sie seit einer Dekade in einem Dilemma gefangen: Sie braucht die Unterstützung sämtlicher rechter Strömungen; mit ihrem legalistischen und angepassten Kurs vergraulte sie jedoch Teile der Szene. Der versuchte Spagat zwischen Extremismus und Bürgernähe misslang.

Völlig unbedeutend und kurz vor dem Sterben ist die NPD jedoch nicht. Unabhängig davon, dass die Selbstauflösung der Partei utopisch ist, verfügt Pastörs über einen guten Draht zu „Freien Kameradschaften“, die nach einem gescheiterten Verbotsverfahren, parallel zu 2003, wieder stärker in der Partei aktiv werden könnten. Außerdem gibt es immer noch Regionen, in denen die NPD Anschluss findet. Auch wenn damit keine Angriffe auf die demokratische Grundordnung möglich werden, vergiftet sie das gesellschaftliche Klima in solchen Orten. Zudem entstehen bei Kundgebungen gegen Asylbewerberheime auf Basis der neuen Demonstrationspolitik Anknüpfungspunkte. Dies ist derselbe populistische Nährboden, der in anderen europäischen Ländern rechten Parteien zu Erfolgen verhalf. All dies darf nicht außer Acht gelassen werden.

Allerdings muss auch erkannt werden, dass die NPD meist nur dann auf Zuspruch hoffen kann, wenn ihre Aktivisten nicht unter dem Parteibanner auftreten. Zu ihren Demonstrationen erscheinen meist nur wenige Menschen. Dass die Aktivisten zu denselben Anlässen mit Tarnung toleriert und akzeptiert, offen jedoch blockiert und ausgepfiffen werden, zeigt, dass das Akronym nach wie vor abgelehnt wird.

So abstoßend Hasstiraden von NPD-Mitgliedern und die Hetze gegen Ausländer, Israel und „Passdeutsche“ auch sind und so verständlich es ist, dass viele Bürger darüber erzürnt sind, dass der Partei eine öffentliche Plattform und Finanzierung geboten wird: Ein Scheitern des Verbotsverfahrens würde die Hasstiraden nur verschlimmern, da es die Partei erneut legitimieren würde und sie wieder Anerkennung in der extremen Rechten finden könnte. ${ }^{101}$ Insofern würde der Demokratie ein schlechter Dienst erwiesen.

Auch könnte ein durchgesetztes Verbot in die falsche Richtung wirken und einen Großteil der NPD-Mitglieder in die Arme der Partei „Die Rechte“ treiben, die neben den weit-

100 Zuletzt lassen sich Möllers und Waldhoff im Verbotsantrag doch auf die einzelnen Personen ein, obwohl sie dies vermeiden wollten - vermutlich, um ihre Ausführungen zu verdeutlichen. Gewichtigere Beweise wie zum Beispiel die direkte Übernahme eines Passus aus dem Parteiprogramm der NSDAP (die Hebung der Volksgesundheit) berücksichtigen sie hingegen nicht. Vgl. Jessica Caus, Nazis im Parlament: Die Wesensverwandschaft der NPD zum Nationalsozialismus, unveröffentlichtes Manuskript an der Universität Passau 2014.

101 Vgl. Jan Kursko, Die Blinden und die NPD, in: Blätter für deutsche und internationale Politik 1/2013, S. 78. 
gehend erfolglosen Republikanern als einzige Partei im rechtsradikalen Spektrum verblieben ist. Nicht zuletzt die Ausschreitungen nach den Kommunalwahlen in Dortmund im Mai 2014 lassen zum einen erkennen, dass die NPD keineswegs der einzige problematische Faktor im institutionalisieren Rechtsextremismus ist - das Wahlergebnis deutet vielmehr auf die steigende Popularität „Der Rechten“ im rechten Spektrum und bei den Wählern hin. Ihr Parteiprogramm birgt Potenzial, jene Gratwanderung zwischen extremen Rechten und Politikfrustrierten zu meistern, an der die NPD gescheitert ist. Ein Mitgliederzustrom ist also auch keineswegs wünschenswert.

De facto ist die NPD, politikwissenschaftlich analysiert, von geringer Relevanz - selbst im Neonazi-Lager. Eine Bedrohung geht von rechtsextremen, militanten Splittergruppen aus und nicht von einer marginalisierten und stigmatisierten Partei, die selbst wie auch ihre Strategie so durchlässig wie durchschaubar ist. Gefährlich sind vor allem die „Freien Kameradschaften“, und im Gegensatz zu Parteien sind sie nur schwer zu überwachen. Die jüngsten Entwicklungen lassen die Szene zunehmend verschlossener agieren, vor allem seit immer mehr Informationen über die Anzahl dortiger Spitzel bekannt werden. Dies wird zum Problem für Beobachter: Kameradschaften verschleiern sämtliche Verbindungen und Tätigkeiten; neuen Kontakten wird grundsätzlich misstraut. Spätestens seit der Causa „Corelli“102 wird es für die Verfassungsschützer immer schwerer, V-Leute zu gewinnen.

Von dieser Szene geht aber nicht nur eine Gefahr in Form von Gewalt aus, es geht auch um die gedankliche Beeinflussung von Jugendsubkulturen. Seit der Rechtsextremismus sein abstoßendes Gesicht verloren hat und mit Hip Hop, Graffiti, Techno und der Adaption linkskultureller Elemente Fuß fasst, verläuft die Anwerbung Jugendlicher so leicht wie nie zuvor. In der rechten Subkultur ist die NPD aber lange nicht mehr Wortführerin; die Autonomen Nationalisten haben ihr hier längst den Rang abgelaufen. Auf diesem Felde sind Präventionsstrategien das richtige Mittel.

Der moralische Faktor für ein Verbot der NPD ist allerdings unschwer zu erkennen: In einem Land, das eine solche Bürde der Vergangenheit trägt, ist es mehr als verständlich, dass die Politik nach den Morden des NSU Zeichen setzen möchte. Zudem kann die Partei als materiell verfassungswidrig gelten, weil sie das bestehende politische System ablehnt und die befundene Wesensverwandtschaft zur Ideologie der NSDAP in der Tat gegeben ist. Insofern müsste man ein Verbot befürworten. Ohne Frage kommt Deutschland hier eine beträchtliche Verantwortung zu. Optimistisch betrachtet ist der Zustand der NPD jedoch ein Sieg der Demokratie, der Gesellschaft und des politischen Willens, der viel schwerer wiegt als ein Sieg vor dem Bundesverfassungsgericht. Die Politische Kultur scheint reif für den offenen Umgang mit Rechtsextremisten. Das Grundgesetz wurde zu einer Zeit der Unsicherheit verfasst; nie wieder sollte eine totalitäre Regierungsform auf dem Gebiet der Bundesrepublik installiert werden können. Doch mittlerweile ist dieses Grundgesetz nicht nur die geschriebene Verfassung des Landes - die Bürger leben es und sollten mit einem Randphänomen wie der NPD umgehen können. Rechtsextremisten sind geächtet und stehen am Rande der Gesellschaft - dies sieht man nicht nur am Widerstand, den die Partei aus der Gesellschaft erfährt.

102 Thomas R. alias „Corelli“ war ein V-Mann des Verfassungsschutzes. Er war tief in die Neonaziszene verstrickt und lieferte über eine lange Zeit Informationen über diese an die Behörden. Im NSU-Prozess wurde er als Zeuge vorgeladen. Anfang April 2014 wurde der im Zeugenschutzprogramm stehende V-Mann tot in seiner Wohnung aufgefunden. 
Dennoch ist ein begründeter Verdacht im Verbotsantrag nicht von der Hand zu weisen: Bei der NPD handelt es sich um eine Partei, die der Ideologie der NSDAP nacheifert. In Deutschland besteht seit dem Ende seines dunkelsten Kapitels der Konsens, dass dieses Gedankengut nie wieder Fuß in der Gesellschaft fassen darf. Die Frage ist daher, welche Form der Demokratie gelebt werden soll. Ist der nonkontroverse Sektor schon vor gedanklicher Bedrohung zu schützen, oder geht es darum, einen gesellschaftlichen Missstand mit jenen 5.000 Rechtsextremen aus der Welt zu schaffen, die in der NPD Mitglied sind? Das BVerfG wird mit seiner Antwort auf diese Frage einen Maßstab setzen, der in den nächsten Jahrzehnten die Politische Kultur beeinflussen wird.

Fest steht, dass das Verbot den Rechtsextremismus in Deutschland nicht aktiv bekämpfen, sondern an der Oberfläche kaschieren würde. Das Ende der Partei bewirkt keine Katharsis: Rassismus, Antisemitismus und Islamfeindlichkeit finden sich in Teilen der Gesellschaft wieder - die NPD hingegen steht weit im Abseits.

\section{Verfassungsmäßigkeit der Befreiung des Südschleswigschen Wählerver- bands (SSW) von der Fünf-Prozent-Klausel. Zum Urteil des Schleswig- Holsteinischen Landesverfassungsgerichts vom 13. September $2013^{1}$}

\section{Florian Edinger}

Erneut wurde nach der jüngsten Landtagswahl in Schleswig-Holstein vom 6. Mai 2012 vor dem Landesverfassungsgericht (LVerfG) um die Gültigkeit der Wahl gestritten. Zuletzt hatte das Gericht Teile des Wahlgesetzes im Hinblick auf Überhang- und Ausgleichsmandate für verfassungswidrig erklärt ${ }^{2}$, und die Landtagswahl musste nach nur zweieinhalbjähriger Legislaturperiode auf das Jahr 2012 vorgezogen werden.

Streitpunkt war diesmal die besondere Rolle des Südschleswigschen Wählerverbands (SSW). Er ist als Partei der dänischen Minderheit nach dem Wahlgesetz von der Fünf-Prozent-Klausel befreit. Der Streit deutete sich bereits im Wahlkampf an: Als die Spitzenkandidatin des SSW anbot, sich an einer rot-grünen Koalition zu beteiligen, wurde von Seiten der CDU kritisiert, die dänische Minderheit dürfe nicht als „Zünglein an der Waage“ über die Regierungsbildung entscheiden. ${ }^{3}$ Der SSW zog mit 4,6 Prozent der Stimmen und drei Abgeordneten in den Landtag ein. Der Wahlausgang war insgesamt so knapp, dass SPD und Grüne auf die Koalition mit der dänischen Minderheit angewiesen waren um zu regieren. Erst die drei Mandate des SSW sicherten die Koalitionsmehrheit von einer einzigen Stimme (35 Sitze der Koalition gegenüber 34 Sitzen der Opposition).

Nachdem der Landtag die Einsprüche gegen die Gültigkeit der Wahl zurückgewiesen hatte, wurde Wahlprüfungsbeschwerde beim LVerfG erhoben mit dem Argument, die Teil-

1 Schleswig-Holsteinisches LVerfG, Urteil vom 13. September 2013, Az. LVerfG 9/12; NordÖR 2013, S. 461 ff., S. 463.

2 Vgl. Patrick Horst, Die schleswig-holsteinische Landtagswahl vom 6. Mai 2012: SPD, Grüne und SSW bilden erste Dänen-Ampel, in: ZParl, 43. Jg. (2012), H. 3, S. 524 - 543.

3 Ebenda, S. 528. 